\section{Brightness and retinal locus: Effects of target size and spectral composition*}

\author{
LAWRENCE E. MARKS \\ John B. Pierce Foundation Laboratory \\ and Yale School of Medicine, New Haven, Connecticut 06520
}

Ss gave numerical estimates of brightness for stimuli presented to the foveal and peripheral retina. Experiment 1 showed that the periphery's superior sensitivity to white light is relatively independent of target size. Experiment 2 showed that the periphery is more sensitive than the fovea to violet light, but is less sensitive than the fovea to red light. These results are explicable in terms of differences between rod and cone mediation of brightness.

The apparent brightness of a flash of light depends upon several parameters of stimulation. These include the radiance, spectral distribution, size, duration, and retinal locus of the stimulus, the presence of inducing fields, the size of the pupillary aperture, and the level of light adaptation just prior to stimulation. Complete specification of apparent brightness requires the knowledge of how all the relevant parameters interact.

The present study aimed to examine how brightness varies with retinal locus of stimulation when three parameters are varied: luminance, size, and spectral composition of the target. Jameson (1965) and Marks (1966) have examined how brightness depends upon retinal locus of stimulation in the dark-adapted eye. They found that the relative brightness aroused by a broad-spectrum stimulus of constant intensity was greater in the periphery than in the fovea, particularly at low levels of stimulus intensity. At high-intensity levels the ratio of peripheral to foveal brightness was much smaller.

More recently, the relation between brightness and retinal locus was investigated under several conditions of prior light adaptation (Marks, 1968). For each of four levels of light adaptation between 0.2 and $200 \mathrm{~cd} / \mathrm{m}^{2}$ ( 58 and $88 \mathrm{~dB}$ re $1 / \pi \cdot 10^{-6} \mathrm{~cd} / \mathrm{m}^{2}$ ) the brightness evoked by a target of constant luminance was maximal at the fovea and decreased regularly as the locus of stimulation was made more and more eccentric. The magnitude of this variation increased as the level of light adaptation increased. Furthermore, the ratio of peripheral to foveal brightness was practically independent of the level of target luminance. On the basis of these results, it was suggested that the brightness of a short flash of light presented to the peripheral

* This research was supported by Contract 1:44620-67-C -0017 with the Air liorce Office of Scientific Research. controlled the exposure duration.

The size of the target was varied by placing circular apertures $(0.32,0.64,0.96$, and $1.60 \mathrm{~cm}$ in diam) just in front of the diffuser. With the $S$ 's eye $0.9 \mathrm{~m}$ from the diffuser, these apertures provided visual angles of $0.2,0.4,0.6$, and $1.0 \mathrm{deg}$, respectively. Red fixation lights (0.1 deg of visual angle) were used to provide the appropriate loci of stimulation. For peripheral stimulation, red lights were placed 12 and $20 \mathrm{deg}$ from the target; the target stimulated the temporal retina of the left eye. For foveal stimulation, each of two red lights was placed 1.2 deg lateral from the center of the target.

Procedure. The $\mathbf{S}$ first dark-adapted for $10 \mathrm{~min}$ with red goggles, then for $3 \mathrm{~min}$ in a dark booth. He was instructed to assign to the first stimulus a number that seemed to him appropriate to stand for its apparent brightness. Then, for succeeding stimuli, he was to assign numbers in proportion to the brightness perceived. Only one fixation light (or the pair of lights to provide foveal stimulation) was on for any exposure of the target.

In the course of a session, each of six luminance levels between 0.025 and $1,250 \mathrm{~cd} / \mathrm{m}^{2} \quad(49$ and $96 \mathrm{~dB}$ re $\left.1 / \pi \cdot 10^{-6} \mathrm{~cd} / \mathrm{m}^{2}\right)$ was presented three times to each of the three loci of stimulation. (For this experiment and for those described in Experiment 2, only the last two judgments for each luminance level were included in the results.) Each exposure lasted $0.5 \mathrm{sec}$, and about $20 \mathrm{sec}$ separated successive exposures. The order of presentation was irregular with regard to both luminance and locus of stimulation and was also different for each S. Twelve men served as Ss. Each served in four sessions, one for each of the four target sizes.

\section{Results}

Figure I gives the geometric means of the brightness estimates as a function of luminance for each of the four target sizes. (A value was not computed for the lowest level of foveal stimulation with the smallest target, since that level could not be seen by half of the Ss.) For each size, it is clear that a given luminance evokes greater brightness in the periphery than in the fovea. Note also that the ratio of peripheral to foveal brightness decreases as the level of luminance increases. This finding has been stated elsewhere (Jameson, 1965; Marks, 1966).

For each locus of stimulation, the estimates of brightness determine a curvilinear function in the double-logarithmic coordinates of Fig. 1. To each set of points was fitted a line having the general equation 


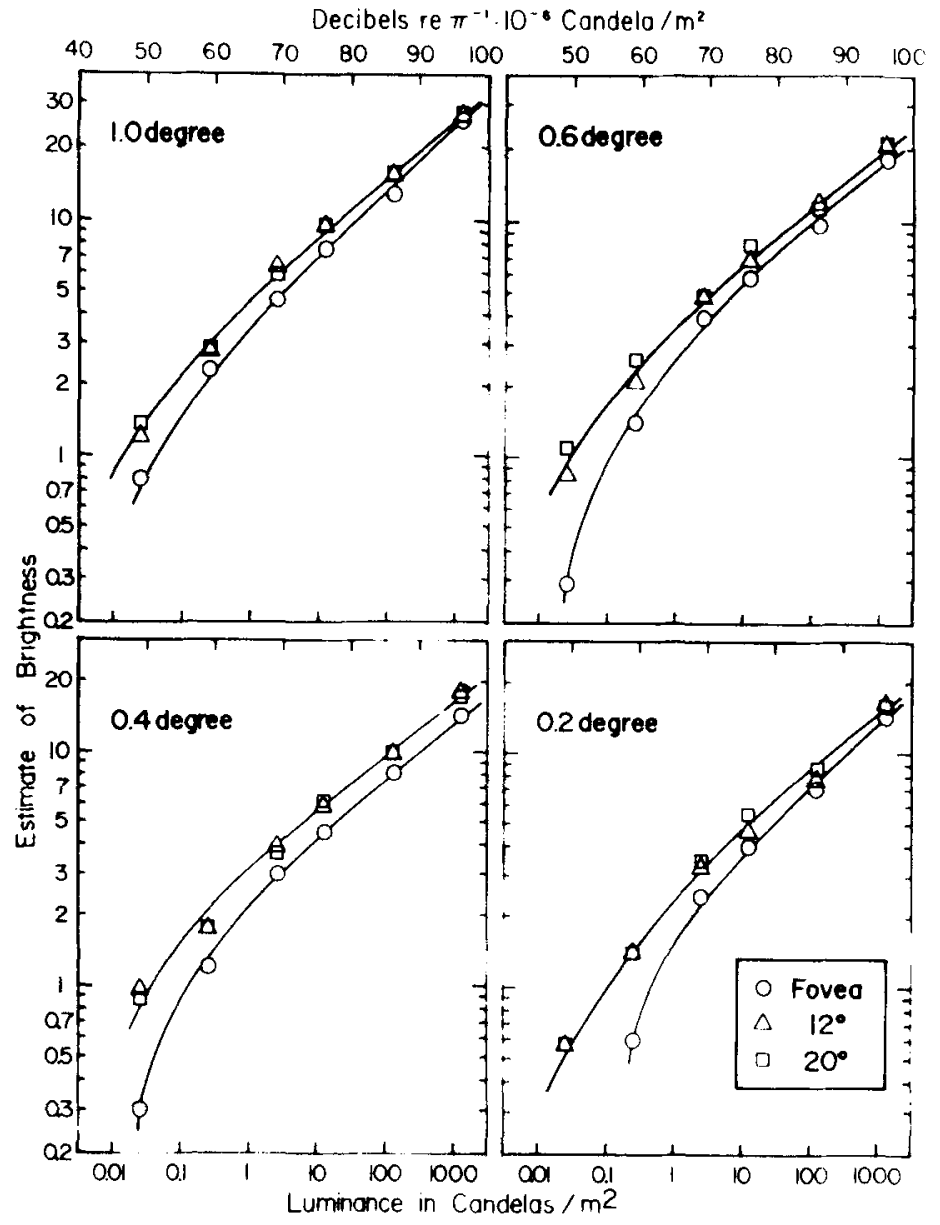

$$
\psi=k\left(\varphi^{\beta}-\mathbf{a}\right),
$$

where $\psi$ is brightness, $\varphi$ is luminance, $a$ is a constant estimated from the data, and $\beta$ is the exponent of the power function. Equation 1 is the psychophysical power law proposed by S.S. Stevens (1961), modified by an additive constant a. (Another modification of the power law relates $\psi$ to $\left(\varphi-\varphi_{0}\right)^{\beta}$. but Eq. I provided a better fit to the present data. For a discussion of the difficulties in deciding between these two modifications, see Marks \& Stevens, 1968.) Since the difference between the brightness produced by stimulation at 12 and $20 \mathrm{deg}$ was generally negligible, a single curve was computed for both peripheral loci. Table 1 gives the values of $\beta, a$, and $k$ for all of the functions.

In fact, the rather marked curvature in the data may well derive from at least two sources. One source is the absolute threshold; curvature in psychophysical functions often occurs in the vicinity of the absolute threshold. Another source is the Broca-Sulzer phenomenon. Use of a $0.5 \mathrm{-sec}$ stimulus duration may have resulted in an "overshoot" in brightness at some levels of luminance. See J. C. Stevens and Hall (1966) and S. S. Stevens (1966). The "correct" description of the psychophysical function for some stimulus durations (e.g., $0.5 \mathrm{sec}$ ) may, therefore, be a rather complex equation to which Eq. 1 can serve as an approximation.

\section{EXPERIMENT 2:}

\section{EFFECT OF SPECTRAL COMPOSITION}

\section{Method}

Apparatus. The basic apparatus was the

\section{Table}

Constants of the Equation $\psi=\kappa\left(\varphi^{\beta}-a\right)$, as Fitted to Estimates of Brightness $(\psi)$ as a Function of Luminance $(\varphi)$ in the Fovea and in the Periphery $(12 \mathrm{Deg}$ and $20 \mathrm{Deg}) . \varphi$ is in $\mathrm{cd} / \mathrm{m}^{2}$.

\begin{tabular}{lllll} 
& Locus & $\beta$ & $a$ & $\kappa$ \\
\hline Size & & & & \\
0.2 Deg & Fovea & 0.21 & 0.57 & 3.5 \\
& Periphery & 0.21 & 0.28 & 3.25 \\
$0.4 \mathrm{Deg}$ & Fovea & 0.20 & 0.41 & 3.7 \\
& Periphery & 0.18 & 0.33 & 4.8 \\
$0.6 \mathrm{Deg}$ & Fovea & 0.22 & 0.38 & 4.2 \\
& Periphery & 0.20 & 0.33 & 5.0 \\
$1.0 \mathrm{Deg}$ & Fovea & 0.25 & 0.245 & 4.5 \\
& Periphery & 0.22 & 0.20 & 5.7 \\
Color & & & & \\
& Fovea & 0.31 & 0.22 & 9.4 \\
Red & 12 Deg & 0.34 & 0.19 & 7.4 \\
& 20 Deg & 0.37 & 0.16 & 20.7 \\
Violet & Fovea & 0.28 & 0.19 & 24.5 \\
\hline
\end{tabular}
and luminance is approximately the same for all wavelengths (Hecht \& Schlaer, 1936). First, for each combination of colored filter plus neutral density used in the magnitude-estimation experiments, the flicker frequency produced by a rotating disk was increased slowly until the $\mathrm{S}$ reported seeing "no flicker." Then, for the lowest frequency that produced a no-flicker response, the luminance of a white target was gradually decreased until

Fig. 1. Geometric means of the magnitude estimates of brightness as a function of luminance (Experiment 1). Retinal locus of stimulation is the parameter. Each section of the figure indicates a different target size.

same as for Experiment 1. Red target light was produced by placing a Wratten No. 70 filter just behind the diffuser. This filter transmits only wavelengths greater than about $650 \mathrm{~nm}$. Violet light was produced by a Corning No. 5.58 filter (maximal transmission about $405 \mathrm{~nm}$ ). The red fixation lights permitted stimulation of the fovea or of loci 12 or $20 \mathrm{deg}$ from the fovea.

Procedure. Two main experiments were run, one with red light and the other with violet light. The $\mathrm{S}$ first dark-adapted for $10 \mathrm{~min}$ in the dark booth. In general, instructions and conditions of viewing were identical to those used in Experiment 1. Different were the number of Ss (14 men in the present experiment), the constant target size ( $1 \mathrm{deg}$ ), and the exact luminances of the six levels used.

Specification of the luminances of the colored targets posed a problem. Heterochromatic matching is often difficult, variable, and dependent upon whether the criterion for a match is equal brightness or minimum border (Boynton \& Kaiser, 1968). The criterion selected for equating luminances was equal $\mathrm{cff}$, since in photopic viewing, the relation between cff 
Decibels re $\pi^{-1} \cdot 10^{-6}$ Candela $/ \mathrm{m}^{2}$

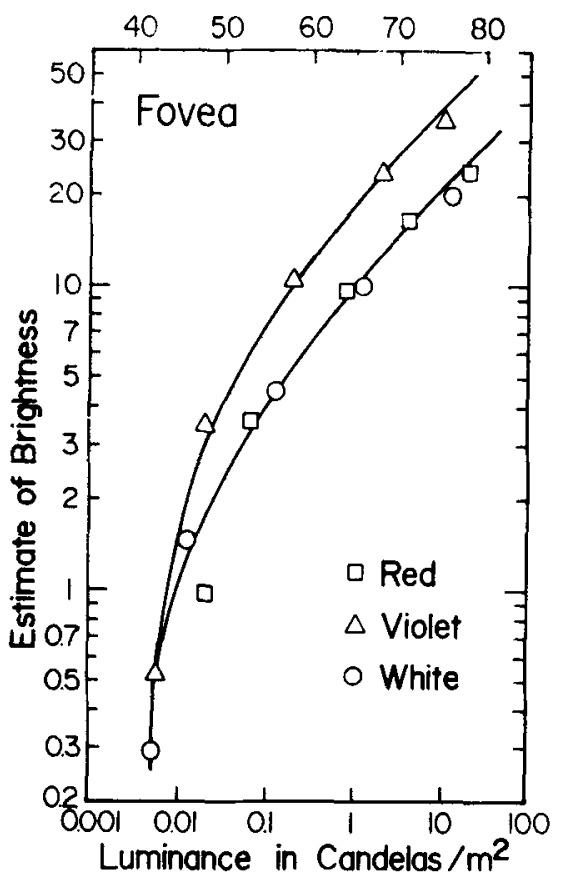

Fig. 2. Geometric means of the magnitude estimates of brightness as a function of luminance of red, violet, and white light presented to the fovea (Experiment 2).

again a response of no flicker was obtained. The average for eight $S s$ of the highest luminance of the white light that produced a no-flicker response was taken to equal the luminance of the colored light. The visual angle of the target was I deg, and $3 . \mathrm{sec}$ exposures of the flickering target were used, with about $20 \mathrm{sec}$ separating successive exposures. Ten minutes of dark adaptation preceded each session.
How luminance measured by this cff technique might relate to judgments of apparent brightness was examined in another experiment. Only the fovea was stimulated, but the six luminance levels of red light and the six levels of violet light, together with six levels of white light, were presented in irregular order in a single session. Instructions and viewing conditions were the same as those of the main experiments, and the same $14 \mathrm{Ss}$ served.

\section{Results}

The geometric means of the brightness estimates for red, violet, and white light (presented foveally) are plotted in Fig. 2. Recall that the levels of luminance shown for the red and violet targets were determined by the flicker-fusion technique described above. Except at the lowest level, the brightness estimates for red light and for white light fall along the same function. In contrast, the brightness estimates for violet light determine a function that lies above that for white and red light. The ratio of violet to red or white brightness is, at any level of luminance, about $2: 1$. Both curves in Fig. 2 conform to Eq. 1.

Figure 3 gives the brightness estimates for red and violet light as functions of locus of stimulation. As the red target is moved from the fovea to the periphery, apparent brightness decreases; as the violet target is moved from the fovea to the periphery, brightness increases. Note that the ratio of peripheral to foveal brightness is relatively independent of luminance level for red light, but is markedly dependent upon luminance level for violet light.

The same geometric means are plotted as functions of luminance in Fig. 4. A line was fitted to each set of points in

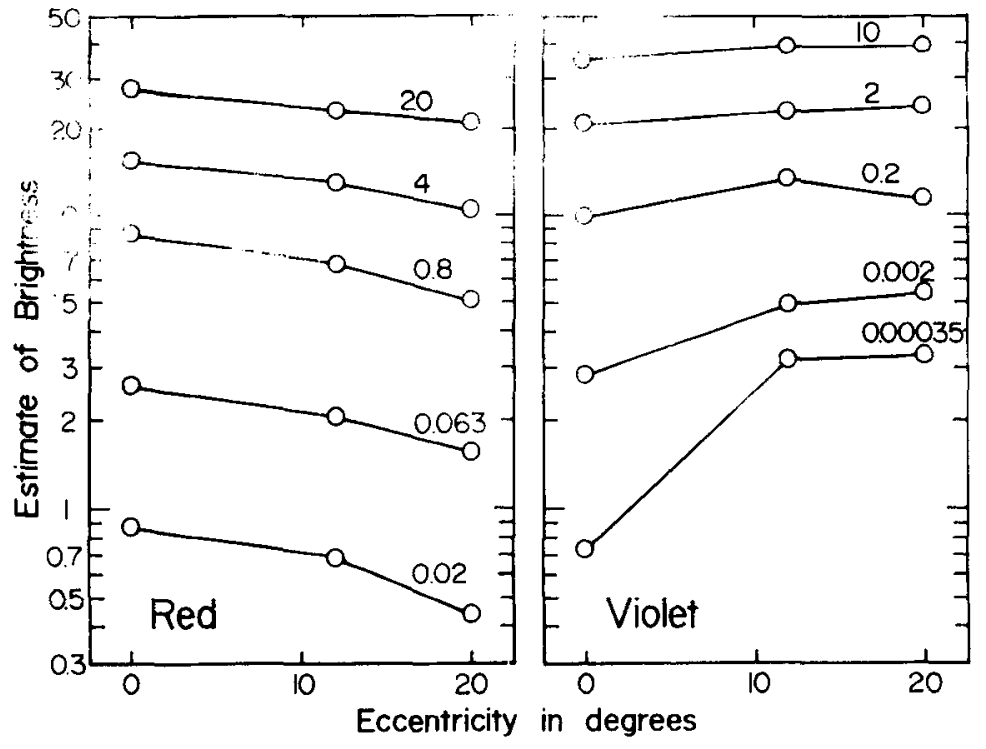

accordance with Eq. 1. (The values of $\beta$, a, and $k$ are given in Table 1.) From these psychophysical functions it was possible to generate equal-brightness contours. These contours, which are given in Fig. 5 , show how luminance must vary across the retina in order to maintain constant apparent brightness. The luminance of a red target presented to the periphery must be greater than the foveal luminance in order to produce the same brightness; in logarithmic terms, the size of this luminance difference is approximately independent of brightness level. On the other hand, the luminance of a violet target presented to the periphery must be lower than the foveal luminance in order to produce equal brightness. Furthermore, this luminance ratio varies systematically with brightness level: a small $\log$ difference separates foveal and peripheral luminances at the highest brightness level, but a larger log difference separates them at the lowest level. Generally similar results were obtained by Bartlett, Sticht, \& Pease (1968) in a study of reaction time.

\section{DISCUSSION}

For every target size examined (0.2-1.0 deg of visual angle) peripheral stimulation with white light produces greater brightness than foveal stimulation (Experiment 1). In Fig. 1 it can be seen that the relation between brightness and retinal locus was not exactly the same for each target size. However, the effect of target size was not systematic: on the average, the 1-deg target gave the smallest ratio and the $0.4-\mathrm{deg}$ target the largest ratio of peripheral to foveal brightness.

The problem of how the relation between brightness and retinal locus depends upon target size is closely related to the problem of spatial summation of brightness. At threshold spatial summation occurs substantially both in the fovea and in the periphery (Graham, Brown, \& Mote, 1939). On the other hand, for brightness levels well above threshold, Diamond (1962) found little or no evidence for spatial summation in the fovea. In order for target size to affect the relation between brightness and retinal locus, it would be necessary that the degree of spatial summation differ from one retinal locus to another.

The effect of spectral distribution on the relation between brightness and retinal locus (Experiment 2) is explicable in terms

Fig. 3. Geometric means of the magnitude estimates of brightness as a function of retinal locus of stimulation (Experiment 2). The luminance of the target $\left(\mathrm{cd} / \mathrm{m}^{2}\right)$ is the parameter. 


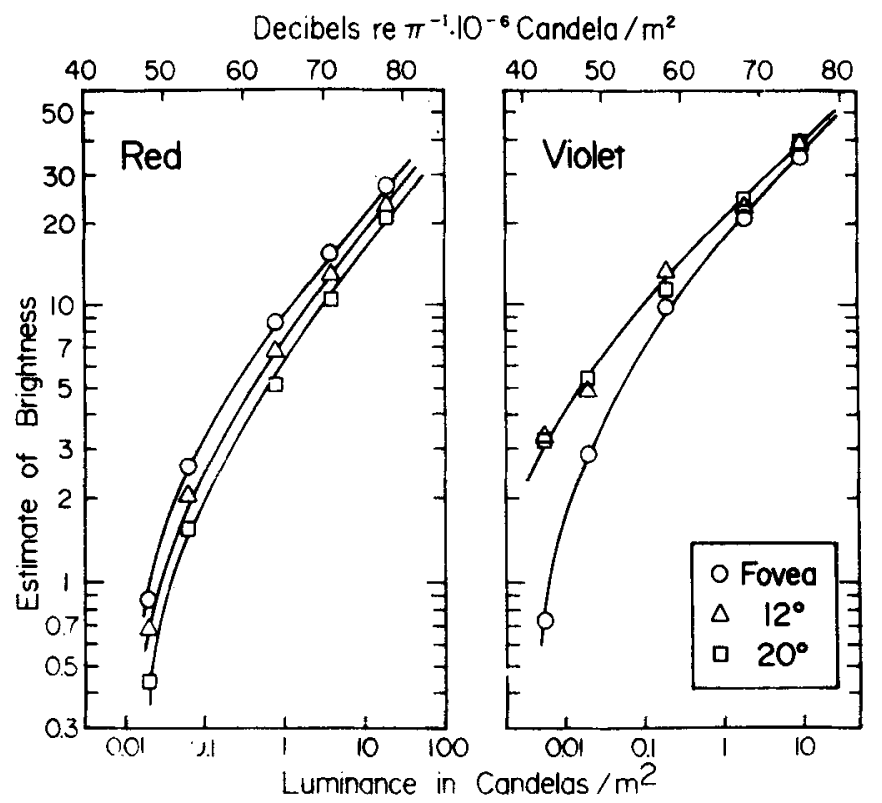

of rod-and-cone mediation of brightness. When a short flash of violet light is presented to the dark-adapted eye, the brightness response is mediated by the cones if stimulation is foveal, but is mediated primarily by the rods if stimulation is peripheral. (The extent of rod mediation would obviously depend upon luminance.) Superior sensitivity of rods over cones could thus account for the greater brightness in the periphery. Other possibilities are the absorption of blue light by the macular pigment (Wald, 1945) and the superior sensitivity to blue light of peripheral over foveal cones (Stiles \& Crawford, 1934). The increase in the ratio of peripheral to foveal brightness at lower luminances may very well relate to the
Fig. 4. The geometric means from Fig. 3 plotted as a function of luminance. Retinal locus is the parameter.

inferior sensitivity of the peripheral retina to red light. Furthermore, to the extent that there is little or no tendency for many exirafoveal cones to converge on single bipolars, the ratio of peripheral to foveal brightness for red light should not depend significantly upon luminance level. This was precisely the result.

Finally, it should be noted that the greater effectiveness of red light in the fovea shows up in other kinds of experiments (S.S. Stevens, 1934). When red and its complementary blue-green are mixed in the proper proportions on a color wheel, the result is a neutral gray. Two striking effects may then be observed. When the $\mathrm{O}$ moves his fixation away from the center of the color wheel, the mixture turns blue-greenish. When he fixates the center, but moves closer to the color wheel, the mixture also turns blue-greenish. If, however, he moves away from the wheel, it turns reddish. Thus the greater relative sensitivity of the fovea to red may show itself when either the size or the location of the retinal image is changed.

\section{REFIRENCES}

BARTLLTT, N. R.. STICHT, T. G.. \& PI:ASE, V. $P$. liffects of wavelength and retinal locus on the reaction time to onset and offect stimulation. Journal of Fxperimental Psychology. 1968. 78, 699-701.

BOYNTON, R. M., \& KAISI:R, R. K. Vision The additivity law made to work for hetcrochromatic photometry with bipartitc ficids. Science, 1968, 161, 366-368.

DIAMOND, A. L. Brightness of a liekd as a function of its area. Journal of the Optical Socicty of America, 1962, 52.700-706.

(;RAHAM, C. H, BROWN, R, H.. \& MOTI, 1 . A. JR. The relation of size of stimulus and intensity in the human eyc. I. Intensity thresholds for white light. Journal of lixperimental Psychology, 1939, 24, 555-573.

HI:CHT, S., \& SCHLAER, S. Intermittent stimulation by light. $V$. The relation between intensity and eritical frequency for ditferent parts of the spectrum. Journal of ciencral Physiology. 1936, 19, 965-979.

JAMISON, D. Threshold and supra-threshold relations in vision. In: Procecdings of the International Colour Meeting: Luccrne. Vol. I. Ciötingen: Musterschunidt-Verlag. 1965. P). 128-136.

MARKS. I.. I . Brightness as a function of retinal focus. Perception \& Psychophysics, 1966. 1 335-341.

MARKS. 1. 1. Brightness as a function of retinal locus in the light-adapted eyc. Vision Rescarch. 1968, 8, 525-535.

MARKS, 1.. I.. \& STEVI:NS, J. C. The form of the puychophysical function near threshold. Pcrciption \& Psycloophysics. 1968. 4. 315-318

Fig. 5. Equal-brightness contours for targets viewed at three retinal loci. These curves are based on the curves in Fig. 4. 
POL.YAK. S. L. The retina. Chicago: University of Chicago Press. 1941.

STl:VliNS, J. C., \& HAL.1., J. W. Brightness and loudness as functions of stimulus duration. Perception \& Psychophysics, 1966. 1, 319-327.

STI:VI:NS. S. S. The relation of saturation to the size of the retinal image. American Journal of Psychology, 1934, 46, 70-79.

STl:VI:NS, S. S. The psychophysics of sensory function, In W. A. Rosenblith (Ed.), Sensory communication. New York: Wiley, 1961. Pp. 1-33.

STl:VENS, S. S. Duration, luminance, and the brightness exponent. Perception \& Psychophysics, 1966, 1, 96-100.

STILES, W. S., \& CRAWIORD, B. H. The liminal brightness increment as a function of wavelength for different conditions of the foveal and parafoveal retina. Procectings of the Royal Society (London), 1934. 113B. 496-530.

WALD, G. Human vision and the spectrum. Science, 1945, 101, 653.658.

WALTERS, H. V., \& WRIGHT, W. D. The spectral sensitivity of the fovea and the extrafovea in the Purkinje range. Proceedings of the Royal Society (London), 1943, 131B, 340-361.

(Accepted for publication April 24. 1970.) 